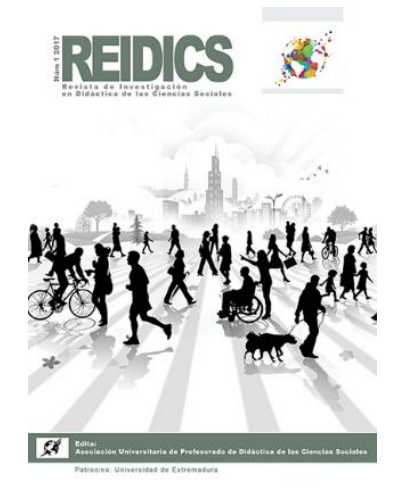

\title{
REIDICS
}

Revista de Investigación en

Didáctica de las Ciencias

Sociales
Núm. 9, 2021

Recibido 01 agosto de 2021

Aceptado 27 setiembre de 2021

E-ISSN: 2531-0968

\section{Étudier les problèmes sociaux-environnementaux: une urgence et un defi pour l'enseignement ${ }^{1}$}

Studying social-environmental issues: an emergency and a challenge for education

\author{
François Audigier \\ Faculté de psychologie et des sciences de l'éducation, Université de Genève, Suisse \\ Email:francoisaudigier@orange.fr \\ ORCID: https://orcid.org/0000-0001-9485-1911
}

DOI: https://doi.org/10.17398/2531-0968.09.41

\begin{abstract}
Résumé
Cet article a pour projet d'exposer certaines des principales caractéristiques de l'étude des problèmes socio-environnementaux (PSE) et d'envisager des transformations nécessaires de nos enseignements dans les disciplines de citoyenneté, géographie et histoire. Dans un premier temps l'auteur décortique un article de presse traitant de la culture bio des tomates en Andalousie. Cet examen est un moyen d'identifier les principaux matériaux et les principales composantes présentes dans un texte qui traite d'un PSE. Certains de ces matériaux et de ces composantes sont appelés à être définis comme intentions d'apprentissage. Dans un troisième temps, il croise les uns et les autres avec les nouvelles demandes sociales et les exigences d'une étude des PSE qui soient à la hauteur des défis que pose l'avenir de la Planète et de l'Humanité. Cela ouvre à un déplacement de nos conceptions des savoirs scolaires et des disciplines qui en organisent la transmission et à la recherche de nouveaux dispositifs d'enseignement. Ce déplacement fait place à la prise en compte explicite des représentations que les élèves ont du monde et du rôle de l'expérience dans la construction des connaissances. Entre ces deux développements, s'insère quelques observations critiques de certaines de nos croyances et conceptions de l'avenir que sont le progrès, la dichotomie nature/société et l'individualisme.
\end{abstract}

Mots clés: problème socio-environnemental; dispositif d'enseignement; apprentissage; sciences sociales; demandes sociales.

\footnotetext{
${ }^{1}$ La plupart des observations de cet article concerne l'enseignement et l'apprentissage dans les disciplines scolaires qui étudient les sociétés présentes et passées - citoyenneté, géographie, histoire - et sont issues de recherches menées en France et en Suisse romande dans le primaire et le secondaire.
} 


\begin{abstract}
The project of this article is to expose some of the main characteristics of the study of socioenvironmental problems (SEP) and to consider the necessary transformations of our teaching in the disciplines of citizenship, geography and history. First, the author dissects a press article dealing with organic tomato cultivation in Andalusia. This exam is a way to identify the main materials and the main components present in a text that deals with an SEP. Some of these materials and components are called to be defined as learning intentions. Thirdly, it intersects both with new social demands and the requirements of a SEPstudy that is up to the challenges posed by the future of the Planet and Humanity. This opens up a shift in our conceptions of academic knowledge and the disciplines that organize its transmission and the search for new teaching devices. This shift gives way to the explicit consideration of the representations that students have of the world and the role of experience in the construction of knowledge. Between these two developments, there are some critical observations of some of our beliefs and conceptions of the future: the progress, the nature/society dichotomy and individualism.
\end{abstract}

Keywords: socio-environmental problema; teaching device; learning; social sciences; social demands.

\title{
1. Introduction
}

L'étude des Problèmes Socio-Environnementaux (ci-après PSE) fait l'objet d'une demande très forte émanant aussi bien de scientifiques que de nombreux acteurs de la société tels que les Organisation Non-Gouvernementale (ONG). Cela se traduit par des décisions politiques qui s'efforcent de trouver une traduction dans les curriculums scolaires. D'aucuns pourraient prétendre que les PSE ne sont pas totalement une nouveauté dans l'enseignement des disciplines scolaires du monde social ${ }^{2}$ (DSMS) particulièrement en géographie - par exemple : les problèmes de l'eau au Proche-Orient, ou l'avancée du désert au sud du Sahara - et que cela n'invite donc pas nécessairement à de nouvelles réflexions, à de nouvelles pratiques. Mais, la situation de la Planète fait de l'étude des PSE une urgence absolue. D'autant plus que si les dérèglements climatiques dominent la sphère médiatique, la limitation des ressources, nombreuses à être de plus en plus rares, les pollutions diverses, de plus en plus graves et fréquentes, augmentent cette urgence.

Aucun de ces éléments n'est une donnée tranquille et stable. Les débats que connaissent nos sociétés sont intenses et souvent tendus. Les intérêts et les pouvoirs oscillent entre coopérations et oppositions. Les conceptions de la vie commune et les valeurs qui les sous-tendent sont diverses et conflictuelles. Ces observations bousculent nos manières de penser la formation des citoyens et, par-là, les contenus et pratiques d'enseignement, avec, par exemple, l'intégration explicite et systématique de la prise en compte de l'avenir, des nécessités de l'action avec ce qu'elle comporte d'incertitudes, une explicitation des valeurs qui fondent nos choix.

Dans cet article, je tente d'identifier et d'exposer très brièvement et partiellement, certains aspects des transformations nécessaires. Je commence par analyser un article de presse qui

\footnotetext{
2 Par Disciplines Scolaires du Monde Social, je désigne des disciplines scolaires qui ont les sociétés présentes et passées comme objets d'étude, y compris dans leurs relations avec la nature. Dans la plupart des systèmes scolaires des États de l'OCDE, ce sont l'histoire et la géographie qui sont au premier rang, presque toujours présentes à l'école primaire et au secondaire en lien avec l'éducation à la citoyenneté.
} 
expose un cas précis, «la culture de tomates bio dans la région d'Almeria». J'identifie différents matériaux - acteurs, actions, chiffres, catégories pour découper le réel, etc. - qui sont utilisés, dans cet article, pour parler de cette culture. Après un bref rappel de l'importance des demandes adressées aux PSE, j'interroge trois de nos «croyances» - le progrès, la domination de la nature, la victoire de l'individu - qui sont aujourd'hui totalement remises en cause mais qui constituent toujours de puissants héritages dans nos manières de penser notre rapport au monde et de nombreux contenus enseignés. Enfin, je m'appuie sur les mises en cause de la forme scolaire dominante sur laquelle se sont construits nos systèmes éducatifs.

Les transformations appelées par ce nouveau contexte et par les nouvelles demandes sociales, invitent à revisiter la contribution des trois disciplines scolaires du monde social et à inventer de nouveaux dispositifs d'enseignement. Ceux-ci demandent de prendre en compte les expériences que les élèves ont de ce monde. Il convient aussi d'accorder une attention particulière au moment de construction du problème et du thème qui vont être étudiés et à celui, en fin de travail, où les apprentissages réalisés, au moins en principe, sont nommés et explicités.

\section{Un exemple: la culture des tomates bio en Andalousie}

Pour introduire l'analyse d'une partie des problèmes posés par l'étude des PSE et les apports de DSMS, je m'appuie sur un article paru dans un journal français, Le Monde, le 2 septembre 2019, sous la plume du journaliste Stéphane Mandard. Le titre est : «En Andalousie, plongée dans l'enfer des serres de tomates bio ». Je complète cet article par des informations recueillies dans des publications scientifiques, (par exemple, Reigada, Delgado, Pérez Neira et Soler Montiel, 2017). L'auteur a mené une enquête sur place, a rencontré ou tenté de rencontrer nombre d'acteurs de cette culture; il situe son propos dans un espace plus vaste, celui des intrants, produits nécessaires à la culture et surtout celui des débouchés commerciaux que sont principalement les rayons bio de la grande distribution en Europe de l'ouest.

J'inventorie les contenus d'un article de presse parce que c'est d'abord, voire exclusivement, par les médias que les citoyens s'informent et prennent connaissance de ce qui se passe autour d'eux et dans le monde. Il est donc capital de travailler avec les élèves sur ces médias. Les connaissances que les enseignants ont de tel ou tel PSE ne se limite pas à ce type de sources. Toutefois, soyons honnêtes, une grande partie de ce que nous enseignons, notamment sur les PSE, n'est pas une traduction, adaptation, simplification de travaux scientifiques validés. C'est un «mixte» entre des travaux scientifiques, eux-mêmes plus ou moins vulgarisés et, sans doute en premier lieu, ce qui vient des médias, surtout lorsque nous sommes dans l'actualité proche. Nos enseignements sont, en principe, légitimés par la maitrise que nous avons des ressources propres aux disciplines que nous enseignons, à notre formation initiale et permanente, donc aux lectures, visions, écoutes outillées et critiques de ces médias.

\subsection{Une intrigue un titre, un sous-titre}

Le titre, « Andalousie, plongée dans l'enfer des serres de tomates bio», est suivi d'un soustitre «Sous les serres d'Almeria, la production été comme hiver d'aliments bio se fait au prix d'une 
surexploitation des ressources humaines et naturelles». L'article va «raconter» au lecteur une «histoire» en combinant des personnages, des actions, des faits, des évènements... Dès le début, l'auteur de l'article affirme un point de vue et utilise les deux catégories habituelles «humaines et naturelles». Une photo montrant des serres en plastique jusque loin sur l'horizon, illustre ces lieux et permet au lecteur de les «imaginer». D'autres photos accompagnent le corps de l'article. Titre et sous-titre constituent l'«intrigue» qui unifie et donne sens à toutes les informations.

\subsection{Les matériaux qui constituent le texte}

Un premier niveau d'analyse consiste à identifier certains des principaux matériaux avec lesquels le texte est construit.

\subsubsection{Des acteurs, des intentions, des pouvoirs}

Les premiers sont les acteurs. Sont nommés dans l'article, des acteurs individuels: un patron, un responsable syndical, une travailleuse marocaine, un avocat, un écologiste.... Chacun de ces acteurs a des intentions, des opinions, des attentes, une position sociale qui lui confère plus ou moins de pouvoir, des valeurs. Sont également présents des acteurs collectifs: les travailleurs, les migrants, les chercheurs, les journalistes... Eux aussi ont des intentions, des opinions, une position sociale, du pouvoir, des valeurs. Nous avons également d'autres acteurs collectifs qui ne sont plus désignés comme des êtres humains mais comme des «entités», principalement des entreprises puisque ce sont elles qui organisent le monde des tomates depuis la production jusqu'à la consommation: la grande distribution - Carrefour, Auchan... - mais aussi les producteurs réunis dans une «puissante confédération» ainsi que les fournisseurs d'intrants comme Monsanto et Bayer. Ces derniers acteurs constituent des «quasi-personnages», pour reprendre une expression du philosophe Paul Ricœur qui désigne par ce terme le fait que, dans nombre de récits, ces entités sont dotées des mêmes pouvoirs que des individus: elles agissent, elles décident, elles négocient, etc.

Si les acteurs individuels ont une réalité matérielle que chacun peut observer, les autres sont des «constructions sociales» et donc des abstractions. Personne n'a jamais rencontré «les» travailleurs ou «l'» entreprise Bayer dans leur diversité, leur complexité, leur dynamique, leur conception du monde. Ces acteurs sont situés dans une société et un «espace-temps» où se nouent des relations de collaboration, de compétition, de complémentarité, d'oppositions, de dépendance, de domination, de conflits...

\subsubsection{Des actions et des activités agricoles, commerciales, sociales, politiques...}

Culture intensive, exportations, irrigation, pollinisation, cueillette, récolte, pollution..., la liste est longue de ces termes qui renvoient chacun à des actions complexes qui sont effectuées selon des temporalités diverses, dans des espaces plus ou moins spécifiques, avec des acteurs variés. À nouveau, nous avons ici des actions et des pratiques en relation les unes avec les autres et avec des conséquences plurielles. 


\subsubsection{Des échelles sociales, spatiales, temporelles}

Les producteurs d'Andalousie dépendent des consommateurs du nord de l'Europe. Pour consommer des tomates, ces derniers dépendent des producteurs. «L'autoroute de la Méditerranée» sillonne entre les serres, direction l'Allemagne, la France, le Royaume-Uni. Produits agricoles dans un sens, revenus monétaires dans l'autre. À ces routes du commerce s'ajoutent les routes empruntées par certains acteurs, notamment les migrants, sans oublier les produits nécessaires à cette agriculture. L'auteur de l'article nous décrit et nous désigne des lieux à différentes échelles: une région, un village, Calahonda, encerclé par des centaines de serres, une ville, Almeria, une partie de l'Europe. Ces échelles spatiales sont aussi liées à des échelles sociales, des individus aux collectifs, et à des échelles temporelles, les temps de production ne sont pas les mêmes que ceux des investissements. Les conséquences écologiques s'inscrivent dans une temporalité différente.

\subsubsection{Milieu, écologie, les données naturelles}

Un autre ensemble de données présentes dans l'article constitue le «milieu» et ce qui relève de l'écologique. En hiver, «les invernaderos du sud-est de l'Espagne tournent à plein régime» tandis que «en trente ans, la biodiversité s'est effondrée». Il y a aussi le problème de l'eau et l'avancée du désert - il faut 50 litres d'eau pour produire un kg de tomates - sans oublier la pollution et la gestion des déchets qui est ici totalement catastrophique.

\subsubsection{Des chiffres, entre quantité, valeurs absolues, pourcentage, valeurs relatives}

Le dernier ensemble que je mentionne concerne les chiffres. Ils sont nombreux et, eux aussi, variées : des quantités absolues, « 108566 tonnes de tomates »; des pourcentages, « 40\% du PIB de la province »; des comparaisons, « l'équivalent de 47.134 terrains de football, trois fois la superficie de Paris ». Or, ces quantités et ces pourcentages sont souvent difficiles à comprendre. Comme avec les terrains de football, les comparaisons sont indispensables.

Ce bref inventaire nomme des « savoirs » qui jouent un double rôle : il faut en maitriser suffisamment pour comprendre l'article ; la lecture et la compréhension de ce dernier est un moment pour en construire de nouveaux. La tâche de l'enseignant est de construire des situations d'apprentissage permettant à l'élève d'occuper ces deux places.

\subsection{Découpage du réel, activités intellectuelles et langages}

Un second niveau d'analyse réunit des composantes plus globales telles que les catégories avec lesquelles nous découpons le réel et les activités intellectuelles en jeu dans les processus de compréhension des situations sociales ainsi que tout ce qui implique les langages.

\subsubsection{Découper le réel}

Les matériaux qui viennent d'être identifiés se rangent dans des catégories avec lesquelles nous découpons le monde et les activités humaines pour les décrire, les analyser, les comprendre. 
Ces catégories structurent très largement aussi bien les champs scientifiques que les rubriques des médias: économie et finance, social et démographie, politique et juridique, culture et religions (plus généralement les croyances)... Elles sont toutes prises en charge par nos disciplines, y compris ce qui relève de la «nature », et sont présentées comme des évidences.

Par exemple, dans ce que raconte l'article, il y a du social, c'est-à-dire des informations qui concernent la santé, le logement avec les bidonvilles (chabolas), les conditions de travail, les cadences, la précarité, les salaires... Ce social prend place dans les relations que les acteurs entretiennent entre eux, notamment de dépendance ou de domination, voire de conflits. Il y a également des informations qui relèvent de l'économie tels que les prix et qui sont en relation avec le social, tels que les salaires, le cout des logements, etc. D'autres ne sont pas nommés explicitement mais demandent à être placés dans des constructions plus vastes, telles que la «chaine de valeurs» en relation avec la chaine de production depuis les intrants jusqu'aux magasins de distribution. Ces chaines constituent autant d'étapes au sein desquelles les acteurs sont dans des relations de collaboration/compétition à la recherche du maximum de gains.

Enfin, je fais une place singulière à la catégorie «politique et juridique» car elle est essentielle pour comprendre, réfléchir, décider et entreprendre. Le citoyen agit dans un cadre donné, celui des lois de la communauté politique dans laquelle il vit, communauté qui se joue ici à plusieurs échelles avec l'Union européenne. Pour engager des actions, élaborer et développer des initiatives, il doit connaitre ce qu'il lui est possible d'entreprendre et comment le faire. C'est une question de libertés, de pouvoirs et de respect du fonctionnement démocratique. Ces normes juridiques sont aussi en relation avec des normes éthiques, autrement dit des valeurs. Les lois ne sont pas nécessairement justes et peuvent être modifiées par l'action politique. Le citoyen est aussi responsable de leur évolution, des changements souhaitables ou redoutés dans les lois.

\subsubsection{Des raisonnements, des activités intellectuelles, des langages}

Dire le monde, les activités humaines, les relations entre les individus, entre les sociétés, etc. se fait sous forme de textes, des textes hétérogènes et aux langages variés. Les matériaux retenus précédemment sont mis en relation les uns avec les autres selon des modalités multiples. Ils décrivent, expliquent, racontent, argumentent le point de vue de leur auteur; ils tissent ainsi des liens avec ce qui, autrement, serait une suite d'éléments étrangers les uns aux autres, un immense bric-à-brac. L'article, comme les manuels scolaires et les documents étudiés en classe, utilisent des langages variés, des langages verbaux, des langages iconiques, des langages linéaires comme le texte lui-même dans sa continuité, des langages spatiaux comme les photos et les cartes.

Ces textes se présentent comme une reproduction plus ou moins exacte du monde. Elles nous font entrer dans la complexité de la vie humaine et sociale ainsi que de nos rapports au monde, mais elles ne sont pas «la» réalité. Il importe d'introduire aussi de la distance critique avec les matériaux qui les composent. Par exemple, à propos des concepts, Koselleck (1990, p. 110) rappelle que «Chaque concept ouvre certains horizons, comme il en ferme d'autres, d'expériences possibles et de théories pensables». Par exemple, qualifier les voitures électriques de voitures «propres» fait croire qu'elles sont une solution décisive dans la lutte contre les dérèglements climatiques. Or, de nombreuses études montrent que, si l'on prend en compte l'ensemble du cycle 
de vie de ce type de voiture, depuis sa production avec les matériaux et l'énergie que demande sa fabrication jusqu'à son démantèlement, y compris avec un recyclage des matériaux, les effets positifs sont loin de s'imposer. Elles ne sont pas «propres» par elles-mêmes. Pour revenir aux «tomates bio», l'article montre très clairement que le «bio» n'est pas nécessairement «écologique». Beaucoup de consommateurs vont penser qu'acheter un légume «bio» est bon pour la Planète; or, si l'on considère l'ensemble du cycle de production et ses effets sur l'environnement, le «bio» s'accompagne, ici, d'une «catastrophe écologique». Nous pourrions ajouter «humaine». De cet exemple, il ne faut surtout pas conclure que toute culture «bio» est contraire à la préservation de l'environnement. Cela implique de travailler avec les élèves sur une diversité d'exemples permettant de poser ces problèmes et d'élaborer des sortes de grilles de lecture de la réalité prenant en compte une pluralité de critères. Cela est aussi un moyen d'entrer dans la complexité des situations socio-environnementales.

\section{Entre nouvelles demandes et croyances}

Dans cette seconde partie, je situe l'étude des PSE sous le double regard des nouvelles demandes qui lui sont adressée et des croyances dont nous héritons.

\subsection{De nouvelles demandes, première aproche}

Il n'est pas besoin d'insister sur les raisons qui poussent de nombreux acteurs à demander que l'École étudie les PSE et forme les citoyens à les prendre en compte dans leur vie personnelle et sociale. Une fois cette généralité énoncée, son analyse montre un très grand nombre de questions et de défis. Si la majeure partie du monde scientifique s'accorde à dire que nous devons faire face à des modifications de notre monde dit naturel, telles que nous devons impérativement modifier nos manières de vivre sur la Planète pour en préserver l'habitabilité, il n'en est pas de même pour de nombreux acteurs sociaux, économiques, médiatiques et politiques.

Vingt ans après le rapport du Club de Rome (1972), le président des États-Unis, George W Bush déclarait «Le mode vie américain n'est pas négociable» s'opposant par-là à la prise en compte des avertissements que nombre de scientifiques adressaient déjà aux autorités politiques. Depuis, nous savons tous que la situation ne fait qu'empirer. Les rapports du Groupe d'experts intergouvernemental sur l'évolution du climat (GIEC) se succèdent, de plus en plus alarmants, sans que les sociétés et les responsables économiques et politiques agissent efficacement pour tenter d'éviter les désastres probables. De nombreuses ressources dites naturelles et exploitées pour produire les biens et services que nous utilisons sont directement menacées. L'étude des PSE devient alors non plus un libre choix laissé aux enseignants, mais une urgente nécessité.

\subsection{Quelques croyances et évolution}

Depuis quelques siècles notre vie en société s'est construite sur plusieurs idées forces qui sont autant de «croyances». J'entends par croyance, le fait que ces idées se sont imposées, d'abord dans nos sociétés occidentales puis à travers le monde, comme des évidences qui dessinaient 
l'avenir et dont la mise en cause est récente: le progrès et la domination de la nature. Ces évidences se sont accompagnées de la construction de sociétés où l'affirmation de l'individu est devenue une valeur suprême. ${ }^{3}$

\subsubsection{Le progrès}

Au cours du Moyen-âge, avec une accélération d'abord lente à partir du XVIII ${ }^{\circ}$ siècle puis de plus en plus forte après la Seconde Guerre mondiale, les sociétés occidentales sont entrées dans une longue période d'augmentation de la production de biens matériels et des activités que leur production et leur usage appelaient. Tout au long de ces siècles, de manière accélérée depuis la fin de la Seconde Guerre mondiale, grâce à la consommation de ces biens, quelques centaines de millions d'individus ont vu leur existence améliorée: fin des famines, meilleure hygiène et augmentation de la durée de vie, ouverture au monde, développement de l'éducation et de la formation, multiplication des marchandises destinées à libérer les humains de nombreuses tâches, etc. Le résultat positif semblait là. Il n'y avait plus qu'à répandre à travers le monde les manières de faire qui assuraient croissance et développement comme facteurs du progrès.

L'idée de progrès subsume le tout, progrès matériels, progrès sociaux, progrès sanitaires... Un seul futur était pensable, celui de l'augmentation continue de la production de biens et de services, unique moyen pour permettre à l'ensemble des humains de connaitre ces améliorations. Après l'Europe et ses extensions (États-Unis, Australie...) et ses capacités à exploiter de nombreuses richesses de la Terre, d'autres États ont poursuivi les mêmes buts. Cela s'est fait par une utilisation massive des ressources de la planète. La prise de conscience de l'épuisement progressif d'un grand nombre de celles-ci, plus encore de la dégradation de la planète due aux activités humaines, met en cause depuis plusieurs décennies cette idée de progrès et ce qui l'accompagne.

Rappelons que si tous les humains vivaient comme les Américains, il faudrait cinq planètes et que, le 31 juillet 2019 (l'année 2020 est trop particulière), nous avons épuisé les ressources disponibles pour une année. Nous pouvons, évidemment, refuser cette réalité et poursuivre notre vie économique, politique et sociale comme si de rien n'était, comme si l'avenir était une simple continuité du présent. Mais... l'étude des PSE ne peut se faire que mise dans cette perspective. Le futur ne saurait être la poursuite tranquille du passé.

\subsubsection{La domination de la nature}

Progrès, croissance et développement se sont diffusés sous l'affirmation d'une distinction rigide entre le monde des humains et le monde de la nature. Les origines de cette séparation sont anciennes, mais la formule de Descartes, souvent citée, exprime clairement l'idée que le développement des connaissances pourra «nous rendre comme maîtres et possesseurs de la nature» (Bonneuil et Fressoz, 2013). Aujourd'hui, il n'est plus possible de concevoir ces relations nature/société en termes d'opposition et de domination. Les travaux se sont multipliés pour les penser de manière complémentaire. L'humain fait partie intégrale de la nature. Si pendant des

\footnotetext{
${ }^{3}$ En écrivant cela, je n'ignore pas que d'autres sociétés, plus holistiques que nos sociétés occidentales individualistes, existent de par le monde.
} 
millénaires, les habitants de la Planète en prélevaient et en exploitaient certains produits, principalement agricoles et miniers, aujourd'hui nos activités dégradent cet habitat commun, des atteintes au climat à l'épuisement de certaines ressources, de la chute de la biodiversité à l'augmentation des inégalités. De nouveaux concepts s'imposent comme celui d' «anthropocène» pour désigner une nouvelle ère où, avec la «révolution thermo-industrielle» $\left(\mathrm{id}^{\circ}\right)$, l'humain agit directement sur la nature et la transforme. John R. McNeill (2010) propose une nouvelle catégorisation du réel qui assure une continuité entre la planète et les activités humaines: lithosphère et pédosphère, atmosphère, hydrosphère, biosphère. Nos catégorisations habituelles deviennent obsolètes. Cela nous invite, nous oblige à penser différemment.

\subsubsection{Le règne de l'individu et le présentisme}

Le troisième élément relève de nos conceptions de la vie personnelle et collective ainsi que de celle du temps qui leur est lié. Ici aussi, les travaux abondent pour décrire la diffusion d'une conception de l'individu qui affirme que c'est lui, lui seul, qui est responsable de ses réussites et de ses échecs. C'est au nom de cette priorité donnée à l'individu que, dans nos sociétés occidentales, les «services publics» ont été en partie privatisés en prétextant leurs couts trop élevés et la déresponsabilisation de chacun. Quant au «présentisme» (Hartog, 2003), ce terme recouvre l'idée selon laquelle nos vies se jouent de plus en plus au présent, qu'il nous faut avoir une réponse à nos questions immédiatement, avoir tout, tout de suite. Par exemple, la diffusion des téléphones portables nous relie directement au monde, aux dernières informations, à des multiples connaissances. Le monde est ainsi devenu immédiatement «disponible» (Rosa, 2020).

Point n'est besoin de longs développements pour insister sur le fait que l'avenir de la Planète et donc de l'humanité est d'abord un problème dont la construction et la solution relèvent du collectif et impliquent la coopération entre les humains. Nos sociétés sont étroitement imbriquées les unes dans les autres. Et pourtant, face à cet impératif, de plus en plus de sociétés, de communautés et de régimes politiques se ferment sur eux-mêmes. Nous devons apprendre aux élèves, aux citoyens à articuler: l'individuel et le collectif, le local et le global, les passés/les présents et les futurs, dans un esprit de solidarité.

Progrès, domination de la nature, importance de l'individu, ont aussi d'autres significations, plus positives, comme en témoignent, par exemple, de nombreuses initiatives qui s'efforcent de penser et de construire un monde différent.

\section{Les PSE, une transformation de nos enseignements}

Un thème tel que «la culture des tomates bio en Andalousie» peut être étudié selon les modalités habituelles de nos enseignements. Le risque est alors d'ignorer les véritables enjeux de l'étude des PSE

\subsection{Un déplacement vital des demandes de formation}

L'étude des PSE rejoint de nombreuses autres demandes de formation souvent réunies sous le terme Éducation à... (Barthes, Lange, Tutiaux-Guillon, 2017) -Éducation à..., à la santé, aux 
médias, à la citoyenneté, au développement durable, à la paix, à l'esprit d'entreprendre, au risque, aux valeurs...-. Les citoyens sont invités à transformer leurs pratiques, donc leurs conceptions de la vie en société, et à œuvrer en conséquence. Le but de l'enseignement n'est plus de former les élèves à résoudre des exercices et des problèmes scolaires dont on attend que les savoirs ainsi maitrisés se traduisent dans leur vie, mais de construire des compétences sociales, c'est-à-dire des compétences pour décider des modalités individuelles et collectives de la vie et agir en société.

Afin de faire toute sa place à la préparation à la décision et à l'action, ceci dans le cadre d'un débat démocratique, je retiens:

- La «prise en compte du futur», non pas un futur qui s'impose comme découlant nécessairement du passé, mais un futur ouvert à l'initiative des citoyens, en fait une pluralité de futurs possibles dont la définition et la réalisation sont sous la responsabilité des citoyens. Cette ouverture implique notamment une réflexion particulière sur les causes de phénomènes et des événements. Nous héritons d'un enseignement, en particulier en histoire, où ces derniers s'enchainent les uns après les autres selon une sorte de nécessité causale, souvent linéaire et simple. Il faut rendre aux passés les moments où d'autres futurs furent possibles et les raisons, notamment humaines, qui ont conduit à faire tel choix et non un autre. ${ }^{4}$ C'est une manière de lutter contre le présentisme et d'introduire de la longue durée.

- Une «pensée de l'incertitude et du risque». Si l'accord existe pour attribuer aux actions humaines les modifications négatives voire catastrophiques que connait la planète - les climats, la biodiversité, la rareté croissante de certaines ressources, la pollution... - les manières d'en anticiper les conséquences et de construire les solutions, appellent une pensée de l'incertitude et du risque. Les savoirs à mobiliser sont, pour une part, en construction, instables, souvent débattus de manière superficielle voire manipulatrice dans certains médias. Dès lors, il est indispensable de fixer ceux sur lesquels les scientifiques sont d'accord, ce qui est acquis, et de les distinguer des questions que de nouvelles recherches explorent pour produire de nouveaux savoirs. Il ne faut pas se tromper de débat. Le doute scientifique n'est pas le doute de sens commun.

Incertitude et risque, invention du futur demandent de rompre avec des causalités linéaires et simplistes et d'introduire de la complexité et du raisonnement probabiliste.

\subsection{Un bouleversement de la forme scolaire}

Ces nouvelles exigences de formation imposent de revenir sur un débat fort ancien dans lequel s'opposent, d'un côté les partisans d'une introduction systématique et ordonnée des élèves dans des contenus disciplinaires élaborés, contenus accumulés et transmis par les générations précédentes, de l'autre ceux qui défendent l'idée de plonger les élèves directement dans des situations de vie pour qu'ils en fassent l'expérience et qu'ils construisent des savoirs et des compétences leur permettant d'y faire face.

\footnotetext{
${ }^{4}$ Une source pour ouvrir ces futurs du passé réside dans les travaux de l'histoire «contrefactuelle» qui réexamine certains faits historiques avec la question: que ce serait-il passé si...?
} 
La progressive généralisation de l'École en Occident depuis le XVIe siècle s'est généralement faite selon la première orientation aboutissant à une organisation propre : les élèves, distribués par groupe d'âge, reçoivent un enseignement commun ; les savoirs sont découpés et rangés dans des disciplines distinctes; les années se succèdent avec des curriculums qui définissent des progressions dans chaque discipline au cours d'une même année, entre les années; le temps quotidien et hebdomadaire est fragmenté. Les savoirs enseignés sont réputés être légitimes puisqu'ils seraient la traduction scolaire de ce qui est considéré comme acquis, comme vrai à un moment donné. Ces savoirs, supposés stables, sont déposés dans des manuels selon une présentation censée être compréhensible par les élèves. Une maîtrise des éléments de base des différents domaines de savoirs est jugée essentielle pour la formation du citoyen. À la suite de travaux d'historiens (Chartier, Compère et Julia, 1976), le sociologue Guy Vincent (1980) a formalisé ce modèle sous le terme «forme scolaire». Il correspond, c'est ce qui en fait l'importance, à un mode spécifique de socialisation. L'élève qui apprend à l'École à soumettre sa conduite à des règles, ne le fait pas par crainte ou par soumission mais par un exercice personnel et collectif de la raison. Loin d'être une servitude, les savoirs disciplinaires sont alors le cadre nécessaire à l'apprentissage et à l'exercice de la raison.

De nombreuses critiques lui sont adressées, notamment celle d'enseigner des savoirs trop éloignés de la vie et qui n'ont pas beaucoup de sens pour une partie importante des élèves et ne les préparent pas à leur vie d'adulte. Dans les faits, il y a toujours eu de nombreux arrangements entre cette forme scolaire et la recherche de situations proches de la vie sociale, de la vie des élèves. Les systèmes scolaires oscillent entre un enseignement disciplinaire systématique et un pragmatisme plus ouvert aux particularités des élèves. Mais si l'organisation de l'École reste sous la domination de cette forme, la recherche d'autres modes de socialisation qui fassent place aux exigences du débat démocratique rappelées précédemment est donc un impératif.

Une des références utiles pour penser les liens à réinventer constamment entre les situations étudiées, les dispositifs d'enseignement, l'expérience des élèves et les savoirs comme outils pour penser, comprendre, analyser, décider, agir, est le pédagogue et philosophe John Dewey. Par exemple, il écrit:

«L'éducation obéit à un principe positif quand les jeunes commencent par avoir des occupations actives ayant une origine et une utilité sociales et s'acheminent ensuite vers la compréhension scientifique des matières et des lois impliquées en intégrant à leur expérience immédiate les idées et les faits communiqués par ceux dont l'expérience est plus étendue.» (Dewey, 1975, p. 253).

\subsection{Contenus et dispositifs d'enseignement}

\subsubsection{Les disciplines scolaires}

Les réalités sociales, les PSE, ne sont pas disciplinaires «par essence». Plus encore, les solutions possibles pour résoudre un PSE ne sont pas disciplinaires. Mais, dépasser le sens commun, le monde des opinions, s'appuyer sur de la connaissance, argumenter et raisonner de façon rigoureuse, impliquent de mobiliser des savoirs venant de travaux scientifiques et adaptés aux élèves. Au-delà des débats sur les transformations et reconstructions que ces savoirs 
subissent lorsqu'ils sont introduits à l'École, l'importance des disciplines s'impose. Si l'on suit Goodman (1978) entrer dans une discipline, ses concepts, ses outils, ses questions, c'est entrer dans une certaine manière de construire le monde, et de s'approprier le réel et mettre en question l'illusion de la transparence de l'expérience, mettre à distance le sens commun.

L'étude d'un PSE n'appartient en propre à aucune discipline. La plupart des disciplines scolaires, voire toutes, sont susceptibles d'apporter une contribution à cette étude. Toutefois, deux ensembles disciplinaires s'imposent en priorité: les sciences de la nature et les sciences de la société; même si cette distinction est aujourd'hui critiquée, elle continue à structurer nos enseignements. La contribution de ces dernières ${ }^{5}$ est obligatoire pour l'étude des PSE. Si chacune mobilise les différentes catégories avec lesquelles sont étudiées les sociétés présentes et passées (voir plus haut), chacune le fait avec ses outils et ses problématiques propres.

Par exemple:

- l'éducation à la citoyenneté est une éducation aux pouvoirs ainsi qu'aux droits, libertés et obligations. Le cas des «tomates bio» permet d'introduire aussi bien la liberté de circulation des marchandises en Europe, ce qui est une liberté, que les droits syndicaux et d'action collective pour les travailleurs;

- la géographie étudie le rapport des sociétés avec l'espace en termes de réseaux et/ou de territoires. Le commerce des tomates bio et la chaine de production demandent à être étudiés en termes de réseau, tandis que le paysage autour d'Almeria correspond à un territoire. Il y a aussi le territoire européen comme espace où se déploient des réseaux et le paysage d'Almeria où les activités sont également analysables en termes de réseau;

- l'histoire ouvre à la prise en compte des différentes dimensions du temps, notamment la longue durée ou le travail de périodisation. La transformation des écosystèmes s'effectue sur une autre durée que les campagnes annuelles de production ou l'embauche de nouveaux travailleurs et l'organisation de leur travail.

Comme écrit précédemment, les trois disciplines disent le monde sous forme de textes et en partagent les quatre formes que sont la narration, la description, l'explication et l'argumentation. Enfin, les apports disciplinaires ne se limitent pas aux seuls concepts mais relèvent aussi des raisonnements et des modes de pensée qui sont propres aux sciences sociales.

Nous devons donc résoudre la tension entre les apports disciplinaires et l'étude de situations, de PSE, qui ne sont pas disciplinaires. En référence à une recherche sur l'éducation en vue du développement durable menée à l'Université de Genève, je reprends une problématique qualifiée par les termes de « détour » et de « retour » (Audigier, Fink, Freudiger, Haeberli, 2011). Le détour disciplinaire correspond à une phase où les outils disciplinaires d'origine scientifique et adaptés aux caractéristiques des élèves, sont introduits et travaillés de manière explicite. Le retour demande de mettre en place des situations suffisamment éloignées des pratiques scolaires habituelles pour se présenter à l'élève comme des situations pouvant exister ou existant dans la

\footnotetext{
5 Voir note $\mathrm{N}^{\circ} 2$.
} 
vie courante, par exemple des situations de débat, l'élaboration et la mise en œuvre de projets, etc. Un des enjeux est la capacité des élèves à mobiliser des savoirs construits durant la phase de détour.

\subsubsection{Une transformation des dispositifs d'enseignement-apprentissage}

L'étude des PSE porte aussi en elle la nécessité d'une transformation des dispositifs de travail dans lesquels les élèves sont placés. Il n'y a pas de modèle qui serait, par essence, pertinent ou efficace. La plupart des auteurs insistent sur leur variété. La prise en compte explicite de l'expérience des élèves, le learning by doing, les programmes par compétences, les collaborations entre plusieurs disciplines, etc., dessinent un nouveau cadre. Quels que soient les dispositifs choisis par l'enseignant ou l'équipe d'enseignants, ils s'organisent autour de différents moments. J'en retiens deux qui sont encadrés par le temps de travail proprement dit, un temps de rencontre avec des contenus apportés par l'enseignant, cherchés par les élèves, etc:

- La dévolution du problème, la présentation du thème, la construction de la problématique;

- L'explicitation des apprentissages recherchés que ceux-ci soient des concepts, des modes de pensée disciplinaires, des capacités d'analyse critique, etc.

Avant de préciser quelques aspects de ces moments, j'insiste sur le fait que, en classe, les élèves n'étudient pas «'la' culture des tomates bio en Andalousie». Ils étudient quelques images et cartes, quelques textes d'origine variée, lesquels sont, presque toujours, des extraits de textes plus longs, des vidéos, elles aussi souvent mises en forme pour tenir dans les contraintes de l'organisation de l'École. Même si les élèves font des recherches eux-mêmes, ils sont toujours aux prises avec un nombre limité d'informations qui sont rangées sous le titre général du thème étudié. Chacun sait, qu'à tout moment, d'autres choix, d'autres documents sont possibles.

Il est illusoire de vouloir travailler l'ensemble des thèmes présents dans l'article du Monde. Il faut donc choisir. Cela peut se faire de différentes manières. En premier lieu, il importe de définir une problématique, un thème qui fait question; par exemple: les effets de la culture de la tomate sur le milieu dit naturel; le système "tomates bio d'Almeria» depuis la production jusqu'à la consommation; les rapports sociaux avec les migrations et les conditions de vie des migrants; la contradiction entre la culture bio et ses conséquences écologiques... Les enjeux d'apprentissage dépassent totalement la seule connaissance d'Almeria. Ce sont des savoirs et des compétences utilisables pour étudier d'autres situations proches ou plus lointaines, d'autres modes de culture des tomates, d'autres modes de production dit «bio»...

La problématique choisie établit une sorte de hiérarchie dans les savoirs et compétences qui sont à construire par les élèves. Les plus importants pour étudier le thème avec la problématique choisis sont aussi les plus qualifiés comme intentions d'apprentissage. Selon la problématique retenue, l'introduction du concept d'anthropocène, le maniement d'échelles, la chaine de production, l'identification des acteurs, de leurs intérêts, des rapports et des conflits sociaux, ce sont eux qui sont l'objet d'un travail d'explicitation, voire de comparaison et de mise 
en relation avec d'autres thèmes étudiés par les élèves à d'autres moments de leur cursus... Il y a aussi ce qui pourrait s'appeler des savoirs et des compétences de second rang. Ceux et celles qui vont être convoqués au service de la construction et de l'explicitation des premières sans faire l'objet d'un apprentissage explicite.

La construction de la problématique et son appropriation par les élèves peut résulter d'un travail commun à partir d'un document propre à provoquer des réactions et réflexions chez eux afin d'établir en commun cette problématique. Elle peut aussi prendre la forme d'une courte intervention magistrale, à la fois claire et concise, qui expose «ce que l'on sait aujourd'hui sur... et le/les problème/s que cela pose», sur le thème retenu. Le travail des élèves consistera à approfondir, à chercher des compléments, à comparer avec d'autres situations, à débattre de la situation, à proposer des solutions, à identifier les points de vue et intérêts de différents acteurs, etc.

Le second temps sur lequel je m'arrête est celui de l'institutionnalisation des savoirs, c'està-dire un temps durant lequel, la classe, les élèves et l'enseignant ou les enseignants, identifient et désignent ce qu'il est important d'apprendre. Un temps de retour sur les apprentissages à réaliser avec les remédiations nécessaires. Il n'y a pas de 'spontanéité' de l'apprentissage. Cette identification est particulièrement délicate dans les DSMS car s'il est toujours nécessaire de s'appuyer sur des études de cas. Or, si toute étude porte sur des situations singulières, les apprentissages ne portent pas seulement, voire peu, sur ces cas eux-mêmes, mais sur des outils de pensée plus généraux, des concepts, des attitudes et des modes de pensée dont j'ai souligné plus haut l'importance.

\subsubsection{Et les élèves?}

Tout ce qui précède n'a (évidemment) qu'un seul but: que l'étude des PSE permette à l'élève de se construire comme citoyen conscient des enjeux du futur et actif dans la société. L'évolution profonde de la forme scolaire dominante dans nos systèmes éducatifs, voire l'invention d'une nouvelle forme scolaire doivent aussi s'appuyer sur ce que l'on connait des modalités d'apprentissage des élèves.

Puisque c'est sous la forme de textes que se construisent et se transmettent nos connaissances et conceptions sur l'expérience humaine individuelle et collective, les modèles de compréhension de textes nous apportent de pertinentes références pour penser et organiser les apprentissages. Comme tout individu, la rencontre des sujets, élèves ou adultes, avec ces textes et donc avec les mondes dont ils parlent, s'établit d'abord en fonction de ce que ils connaissent du thème traité, de certains schémas explicatifs des situations sociales qu'ils ont en tête, de leur attitude à l'égard de ce sujet. Ces représentations constituent le premier filtre pour recevoir et interpréter les connaissances nouvelles présentes dans le PSE. La connaissance se construit d'abord par analogie avec ce qui est déjà connu. Puis, les différences avec ce «déjà-là» conduit à prendre de la distance avec ce dernier et à élaborer de nouvelles connaissances, à enrichir ce que l'on sait et comprend du monde. Au cours de ce processus, les attitudes, les positions de l'élève à l'égard de ce qui est enseigné, jouent un rôle très important, facilitant l'adhésion à ce qui est enseigné ou établissant des sortes de refus ou d'adhésion plus ou moins partielle, plus ou moins 
conforme. Ils participent à un monde, à des sociétés, à des cultures qui, à la fois, sont en mobilité constante et informent leurs points de vue. Par exemple, la recherche genevoise montre que les élèves reproduisent, à leur manière, la diversité des points de vue présents dans nos sociétés: scientistes en pensant que les sciences et techniques vont résoudre les problèmes posés; indifférents car n'accordant pas d'importance à ces derniers; individualistes en imaginant qu'ils trouveront eux-mêmes des solutions; autoritaires en appelant les pouvoirs publics à des décisions contraignantes; sceptiques ou résignés avec l'idée d'une vision pessimiste de l'avenir...

En reprenant et en déplaçant quelque peu le terme d'expérience introduit en référence à Dewey, celle-ci joue un rôle déterminant dans l'élaboration de nos connaissances, de notre rapport au monde et aux autres. Cette expérience est à la fois directe et indirecte. Elle est indirecte par ce que nous savons du monde par la médiation des autres et ce qu'ils nous en racontent, les autres individus rencontrés, les médias, les réseaux sociaux, notre entourage, etc. Elle est directe lorsque nous sommes nous-même parties prenantes de telle ou telle situation de tel ou tel évènement. L'une et l'autre sont des éléments essentiels dans l'élaboration de nos représentations sociales.

Dewey appelle à la construction de dispositifs mettant les élèves en situation d'expérience directe. Dans l'enseignement des PSE, tenir compte de l'expérience indirecte se fait de deux manières. La première se base sur le fait que les élèves ont l'expérience, la connaissance de situations qui les informent des problèmes de la Planète, problèmes devant lesquels ils restent indifférents ou qui les touchent et les concernent, selon les cas. La seconde tient au fait qu'ils ont eux-mêmes des pratiques sur lesquelles se fonder pour étudier les PSE et construire des outils, tel le concept de réseau, ou pour réfléchir sur leurs attitudes face à certains agissements personnels ou collectifs.

Le sens des savoirs enseignés réside dans les relations que les individus, ici les élèves, établissent avec leurs expériences, les questions qu'ils se posent à propos des thèmes enseignés, les choix qu'ils pourront faire dans leur vie. Étudier les tomates en Andalousie ne se réduit pas à l'étude d'un thème comme un autre, mais prend totalement son sens avec les conséquences possibles dans sa propre vie, par exemple : puisqu'il y a pollution, destruction de la nature, etc., dois-je encore consommer des tomates produites selon ces modalités ? Les cultures bio ont-elles souvent, toujours, de telles conséquences sur le milieu?

$\mathrm{Au}$ fondement des attitudes et des capacités à réfléchir, à orienter son point de vue et ses actions, se placent les valeurs, en liens avec les croyances et les visions du monde. Selon les priorités accordées à telle ou telle valeur dans une situation donnée, les attitudes et les comportements des individus et des groupes peuvent fortement varier dans le temps ou dans l'espace. Dès lors, il est capital de leur faire une place particulière et explicite dans l'enseignement car, d'une manière ou d'une autre, elles sont déterminantes dans l'établissement de nos jugements, de nos décisions, de nos actions, voire les fondent (Audigier, 1991).

Je précise que les valeurs ne sont pas des «choses» que l'on applique à la manière d'une ordonnance médicale. Elles dessinent un «point de vue» à partir duquel l'individu, les groupes, les collectifs... évaluent les situations et les actions humaines et sociales, en analysent les effets, posent des initiatives, font des projets, etc. Dès que l'on prend une décision, que l'on entreprend 
une action, que l'on étudie ou cherche à comprendre une situation, les valeurs sont là. Elles ne sont pas un monde tranquille; le plus souvent, elles sont en tension voire en conflit les unes avec les autres. Les valeurs auxquelles chacun adhère engagent également ses émotions à travers ses rapports au monde et aux autres.

\section{Trois questions, quatre chantiers}

L'examen de certaines des plus fortes caractéristiques des PSE fait dans ce texte rappelle que tout curriculum, tout choix d'enseignement ouvre trois questions qui débouchent ellesmêmes sur quatre chantiers.

Trois questions:

- Une question politique. Si l'on adhère à l'idée démocratique et au pouvoir des citoyens, l'enjeu est d'ouvrir l'avenir à leur initiative, de former au débat, à leur responsabilité et à celle des pouvoirs.

- Une question cognitive. Pour ouvrir cet avenir, il faut aussi ouvrir les savoirs, c'està-dire admettre la pluralité des interprétations (Citton, 2010) et en faire un objet de travail et d'étude.

- Une question éthique. L'action et la construction du futur, des futurs individuels et collectifs, portées par l'étude des PSE mettent à la question les relations entre les fins et les moyens. Selon Karl Marx cité par Albert Camus dans L'homme révolté (1951), «Un but qui a des moyens injustes n'est pas un but juste».

Devant les enjeux de l'avenir de la Planète et de la construction de notre vie commune, ces trois questions sont à développer en réaffirmant avec force l'indispensable citoyenneté démocratique, une citoyenneté fondée sur les droits humains. L'article premier de la Déclaration universelle de 1948 n'a rien perdu de sa force: «Tous les êtres humains naissent libres et égaux en dignité et en droits. Ils sont doués de raison et de conscience et doivent agir les uns envers les autres dans un esprit de fraternité». L'exigence de fraternité se joint à celle de justice pour accompagner toute étude d'un PSE, toute recherche de solution.

Quatre chantiers:

- La prise en compte de la diversité des savoirs nécessaires aussi bien à l'analyse d'une situation sociale qu'au choix des initiatives et actions à entreprendre.

- L'explicitation critique et la possible modification des catégories et des concepts avec lesquels nous découpons et analysons les sociétés et les activités sociales.

- L'examen de la possibilité d'une organisation de l'enseignement, donc des curriculums, autour de l'étude de situations. Toutefois, à la fragmentation actuelle des savoirs qui est souvent critiquée pour mettre en cause les découpages disciplinaires, il convient de ne pas répondre par la multiplication 
des situations étudiées et des Éducation à... qui risque alors de produire une fragmentation tout aussi critiquable.

- La place et le rôle des savoirs disciplinaires qui sont une contribution essentielle à la formation critique pour problématiser l'étude et la réflexion sur toute situation sociale, donner du sens aux expériences, permettre à chacun de construire des points de vue raisonnés, mettre à distance les discours sur le monde, etc.

\section{Références bibliographiques}

Audigier, F. (1991). Enseigner la société, transmettre des valeurs; former des citoyens, éduquer aux droits de l'homme: une mission ancienne, des problèmes permanents, un projet toujours actuel. Revue Française de Pédagogie, (94), 37-48.

Audigier, F., Fink, N., Freudiger, N. et Haeberli, P. (dir.). (2011). Éducation en vue du développement durable: sciences sociales et élèves en débats. Genève: Université de Genève, Cahiers de la section des sciences de l'éducation.

Barthes, A., Lange, J.-M. et Tutiaux-Guillon, N. (2017). Dictionnaire critique des enjeux et concepts des «éducation à». Paris: L'Harmattan.

Bonneuil, C. et Fressoz, J.-B. (2013). L'événement anthropocène. Paris: Seuil.

Camus, A. (1951). L'homme révolté. Paris: Gallimard.

Citton, Y. (2010). L'avenir des humanités. Paris: Éditions La Découverte.

Chartier, R., Compère M.-M. et Julia, D. (1976). L'Éducation en France du XVIe au XVIIIe siècle. Paris: SEDES.

Descartes, R. (1637-1824). Discours de la méthode. Texte établi par Cousin, V. Levrault, tome I, sixième partie.

Dewey, J. (1975). Démocratie et éducation. Paris: Armand Colin. Traduction de Dewey, J. (1916). Democracy and Education: An introduction to the philosophy of education.

Goodman N. (1978). Ways of Worldmaking. Indianapolis: Hackett.

Hartog, F. (2003). Régimes d'historicité. Présentisme et expériences du temps. Paris: Le Seuil.

Koselleck, R. (1990). Le Futur passé. Contribution à la sémantique des temps historiques. Paris: Éditions de l'École des hautes études en sciences sociales. Traduction de Koselleck, R. (1979). Vergangene Zukunft. Zur Semantik geschichtlicher Zeiten. Suhrkamp.

Mandard, S. (2 septembre 2019). En Andalousie, plongée dans l'enfer des serres de tomates bio. Le Monde. https://www.lemonde.fr/planete/article/2019/09/02/en-andalousie-plongeedans-l-enfer-des-serres-de-la-tomate-bio_5505296_3244.html

McNeill, J. R. (2000). Something New Under the Sun - An Environmental History of the TwentiethCentury World. New York: Norton.

Reigada, A., Delgado M., Pérez Neira D. y Soler Montiel M. (2017). La sostenibilidad social de la agricultura intensiva almeriense: una mirada desde la organización social del trabajo. Ager, (7), 1-26.

Rosa, H. (2020). Rendre le monde indisponible. Paris: La Découverte. Traduction de Rosa, H. (2018). Unverfügbarkeit. Residenz Verlag. 
The club of Rome. (1972). The Limits To Growth. Chelsea: Chelsea Green Publishing.

Vincent, G. (1980). L'École primaire française. Lyon: Éditions de la Maison des sciences de l'homme. 